\title{
Large Schwannoma of the Deep Peroneal Nerve: A Case Report
}

\author{
Baris ERDOGAN ${ }^{1}$, Mehmet O. YUKSEL ${ }^{2}$, Engin TANER ${ }^{3}$, Asli CAKIR ${ }^{4}$ \\ ${ }^{1}$ Sanliurfa Training and Research Hospital, Department of Neurosurgery, Sanliurfa, Turkey \\ ${ }^{2}$ Medipol University, Bahcelievler Nisa Hospital, Department of Neurosurgery, Istanbul, Turkey \\ ${ }^{3}$ Mehmet Akif Inan Training and Research Hospital, Department of Neurosurgery, Sanliurfa, Turkey \\ ${ }^{4}$ Medipol University, School of Medicine, Department of Pathology, Istanbul, Turkey
}

Corresponding author: Baris ERDOGAN dr.baris.erdogan@gmail.com

\section{ABSTRACT}

This article presents the case of a 32-year-old female patient with schwannoma. The patient had swelling on the anterior aspect of her right foot for 1 year with increasing pain over the past 2 months. Moreover, a positive Tinel sign was present over the swelling. Magnetic resonance imaging revealed a large schwannoma mass in the deep peroneal nerve. Consequently, the patient's large schwannoma was completely excised along with its capsule.

Schwannomas are benign tumors of the peripheral nerves that rarely exhibit malignant transformation. Treatment is considered to be curative if complete resection is achieved.

KEYWORDS: Edema, Magnetic resonance imaging, Pain, Peroneal nerve, Schwannoma

ABBREVIATIONS: MRI: Magnetic resonance imaging, NF: Neurofibromatosis, US: Ultrasound

\section{INTRODUCTION}

S chwannomas are the most common benign tumors of the peripheral nerves $(80 \%)$, originating from the Schwann cells which manufacture the myelin sheath around the axons in the peripheral nerves (4). They occur most often between the third and sixth decades and exhibit no gender and ethnicity preponderance. Schwannomas were first described by Varocay in 1908, which constitutes $5 \%$ of all soft-tissue tumors (3). They are often noted in the head and neck, flexor surface of the extremities (especially, the ulnar and peroneal nerve regions), mediastinum, and retroperitoneum but they can occur in any region of the body (14).

Schwannoma is mostly seen as a single lesion. However, multiple tumors can be observed in schwannomatosis and neurofibromatosis type 2 (10). Schwannoma is less aggressive and has a much better prognosis than malignant peripheral nerve sheath tumors (6). Moreover, schwannoma allows perfect enucleation due to its good cleavage pattern (9).

The histopathological findings of schwannomas reveal a biphasic pattern with Antoni-A and B areas. Antoni-A areas have highly organized spindle cells, short parallel fascicles, and less onion-like or swirl arrangement. In contrast, Antoni-B domains are less cellular with myxoid tissue predominance and water-like fluid filling abundance in cytosols and extracellular space. The target sign was defined as the presence of central and peripheral Antoni-A B areas, respectively. Specifically, schwannomas are S100 protein-positive (15).

Most patients may have swelling, localized mass in the nerve area, pain, numbness, and neurological deficits at the time of presentation. Moreover, ultrasound (US) and magnetic resonance imaging (MRI) can be used to diagnose schwannoma. Peripheral nerves are defined as hypoechoic
Baris ERDOGAN (D): 0000-0003-4946-0748 Mehmet O. YUKSEL (1) : 0000-0003-0922-4249
Engin TANER (1) : 0000-0003-4810-0943

Asli CAKIR (1D) : 0000-0003-0128-6947 
tubular structures around the epineurium during ultrasonic examinations. However, the continuity of this tubular structure is disrupted in the proximal and distal areas in the presence of a tumor in the peripheral nerve. US plays a role in the differential diagnosis of peripheral nerve tumors and lesions such as lymphadenopathy, hematoma, abscess, and thrombosis (13). T1, T2, and contrast-enhanced sequences in $\mathrm{MRI}$ are diagnostically useful because it facilitates diagnosis in $75 \%$ of cases. Moreover, the sagittal and coronal images help delineate the entry and exit points of the tumor (8). The mass signal is notably hypointense or isointense on T1-weighted images and hyperintense or heterogeneous on T2-weighted images owing to the intrinsic changes in the tumor.

Nevertheless, the treatment criteria do not only depend on the tumor size but also the patient's condition and clinical findings. Surgery is not indicated in asymptomatic patients based on tumor size alone. However, surgery is indicated in patients with progressively increasing tumor volume, pain, paresthesia, and progressively worsening neurological deficits. Thus, surgical treatment aims to notably preserve nerve continuity and structural integrity and completely remove the tumor capsule before the development of neurological deficits (1).

\section{CASE REPORT}

A 32-year-old female patient had swelling on the anterior aspect of her right foot for 1 year with severe pain over the past 2 months with positive Tinel over the swelling. An MRI was done upon presentation to the outpatient clinic with these complaints. A mass lesion (size, $68.5 \times 26.5 \times 15.5 \mathrm{~mm}$ ) was observed with smooth and lobulated contours, located at the deep peroneal nerve between the tibialis anterior and extensor hallucis longus tendons. Postcontrast examinations revealed an intense contrast enhancement (Figure 1). The patient's large schwannoma located in the deep peroneal nerve was completely excised along with its capsule (Figure 2A). Consequently, pathological examination confirmed the mass as schwannoma (Figure 2B-D).

This study followed the ethics rules of the 1964 Declaration of Helsinki, which was amended in 2013. Moreover, informed consent was obtained from the participant.

\section{DISCUSSION}

Schwannomas, also known as neurilemmoma, neuroma, neurilemmoma, and Schwann cell tumors are benign tumors of the peripheral nerve sheath. They often occur between the third and sixth decades and exhibit no preponderance related to gender or ethnicity. They can occur at various locations in the body (e.g., upper and lower extremities, mediastinum, and retroperitoneum).

Schwannomas exhibit a slow-growing pattern and their malignant transformations are rare. They cause various clinical symptoms. The patient may not have any complaints or have several complaints (e.g., cosmetic deformity, palpable mass, pain, paresthesia, numbness, and neurological deficits) at the time of presentation. Before deciding on the treatment method, the patient's condition, neurological findings, and radiological findings should be evaluated. However, surgery is the primary treatment method.

The surgical treatment aims to preserve the nerve integrity and continuity, as well as completely excise the mass (9). Several risk factors have been reported regarding the complications and development of postoperative deficits. The risk of complications and postoperative neurological loss has been reported to be higher in young patients with a large volume, proximal location, ulnar nerve location, and major motor nerve location. The literature review reveals reports by Raj et al. (67\%), Mizushima (46\%), and Hirai (35\%) who have stated that deficits

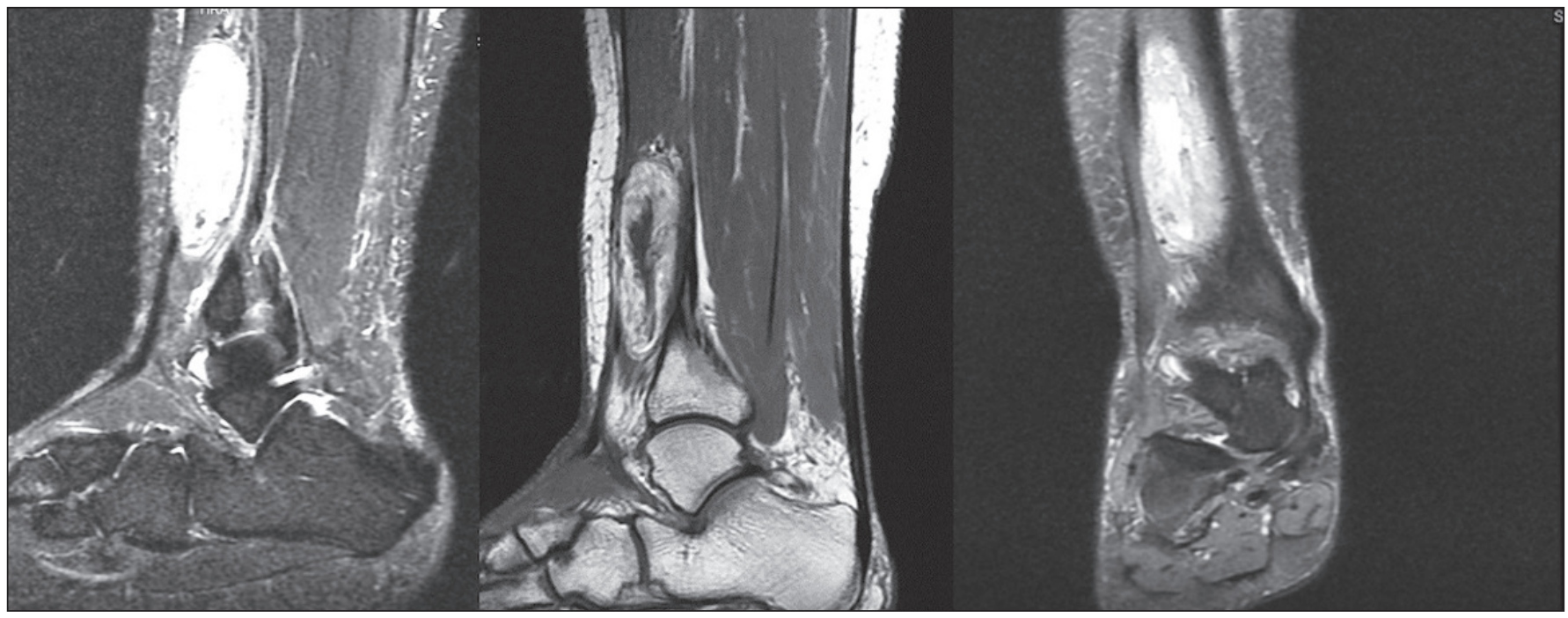

Figure 1: Magnetic resonance imaging examination with sagittal STIR sequence, contrast sagittal T1 sequence, and coronal plane fatsuppressed sequence. A mass lesion of $68.5 \times 26.5 \times 15.5 \mathrm{~mm}$ was observed that had smooth and lobulated contours and was located near the deep peroneal nerve between the tibialis anterior and extensor hallucis longus tendons. Moreover, postcontrast examinations revealed intense contrast enhancement. 

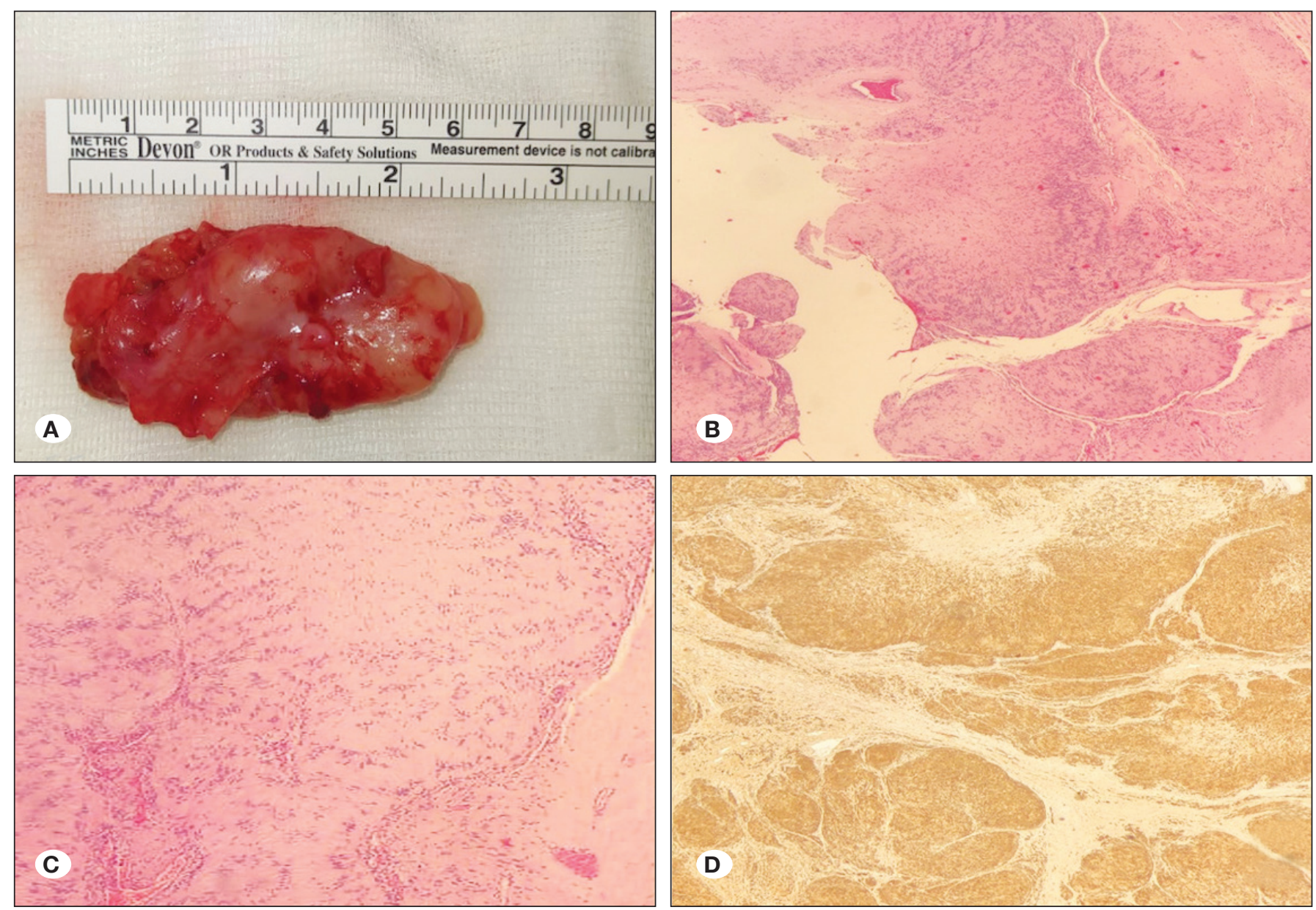

Figure 2: Macroscopic and microscopic pathological views. A) Macroscopic view of totally excised schwannoma. B) Spindle cell tumor with cellular and hypocellular areas. Vessels with the thickened hyalinized wall can be observed (HE $\times 40)$. C) Palisaded nucleus of tumor cells forms Verocay bodies $(\mathrm{HE} \times 100)$. D) Diffuse nuclear and cytoplasmic S100 expression in tumor cells $(\mathrm{S} 100 \times 40)$.

may worsen during the postoperative period (2, 5, 9). However, Raj et al. reported that sensory deficits (e.g., paresthesia or hypoesthesia) improved during the postoperative period (9). They stated that tumor size, surgical technique, and use of electrophysiological monitoring according to the operation are crucial in preventing neurological deficits (9). However, despite all techniques, tumor enucleation is impossible which is sometimes due to perifascicular invasion. In such cases, major neurological deficits may occur because of wider excision (e.g., fascicular resection). Sawada et al. reported that permanent neurological deficits developed because of fascicular resection in four brachial plexus cases (11).

A literature review on peroneal nerve schwannoma reveals that most publications are in the form of case reports. Case reports published by Nascimento et al. and Shariq et al. reported that the main treatment principle was complete resection $(7,12)$. In addition, treatment has been notably curative in cases that underwent complete resection.

\section{CONCLUSION}

In conclusion, schwannomas are benign tumors of the peripheral nerves that rarely exhibit malignant transformation. Nevertheless, jointly evaluating the patient's clinical condition and the lesion's characteristics is crucial to determine the most appropriate treatment modality. Moreover, the use of additional assistive and monitoring tools during surgery could help prevent complications. Nonetheless, treatment is considered to be curative if complete resection is achieved.

\section{REFERENCES}

1. Desai KI: The surgical management of symptomatic benign peripheral nerve sheath tumors of the neck and extremities: An experience of 442 cases. Neurosurg 81:568-580, 2017

2. Hirai T, Kobayashi H, Akiyama T, Okuma T, Oka H, Shinoda Y, Ikegami M, Tsuda Y, Fukushima T, Ohki T, Ishibashi Y, Sawada R, Goto T, Tanaka S: Predictive factors for complications after surgical treatment for schwannomas of the extremities. BMC Musculoskelet Disord 20:166, 2019 
3. Jonathan CK, Fu-Keung IP, Tak-Chuen W, Prisilla L, SzeYan C: Neurilemmomas of the hand: a review of the clinical presentation, surgical outcome and ptential risk factors. Othop 22:48-51, 2017

4. Kang HJ, Shin SJ, Kang ES: Schwannomas of the upper extremity. J Hand Surg Br 25:604-607, 2000

5. Mizushima $\mathrm{H}$ : Neurological deficits before and after surgical resection of schwannomas in the upper extremities. J Reconstr Microsurg 32:371-377, 2016

6. Montano N, D'Alessandris QG, D'Ercole M, Lauretti L, Pallini R, Di Bonaventura R, La Rocca G, Bianchi F, Fernandez E: Tumors of the peripheral nervous system: Analysis of prognostic factors in a series with long-term follow-up and review of the literature. J Neurosurg 125:363-371, 2016

7. Nascimento G, Nomi T, Marques R, Leiria J, Silva C, Periquito $\mathrm{J}$ : Ancient Schwannoma of superficial peroneal nerve presenting as intermittent leg pain: A case report. Int J Surg Case Rep 6C:19-22, 2015

8. Nilsson J, Sandberg K, Soe Nielsen N, Dahlin LB: Magnetic resonance imaging of peripheral nerve tumours in the upper extremity. Scand J Plast Reconstr Surg Hand Surg 43:153159, 2009
9. Raj C, Chantelot C, Saab M: Predictive factors of postoperative deficit and functional outcome after surgery for upper limb schwannomas: Retrospective study of 21 patients. Hand Surg Rehabil 39:229-234, 2020

10. Rouleau GA, Wertelecki W, Haines JL, Hobbs WJ, Trofatter JA, Seizinger BR, Martuza RL, Superneau DW, Conneally PM, Gusella JF: Genetic linkage of bilateral acoustic neurofibromatosis to a DNA marker on chromosome 22. Nature 329:246-248, 1987

11. Sawada T, Sano M, Ogihara H, Omura T, Miura K, Nagano A: The relationship between pre-operative symptoms, operative findings and postoperative complications in schwannomas. $J$ Hand Surg Br 31:629-634, 2006

12. Shariq O, Radha S, Konan S: Common peroneal nerve schwannoma: An unusual differential for a symptomatic knee lump. BMJ Case Rep 2012:bcr2012007346, 2012

13. Valle M, Zamorani MP: Nerve and blood vessels. In: Bianchi S, Martinelli C (eds). Ultrasounda of the Muskuloskeletal System. Springer-Verlag: Berlin, 2007:97-134

14. Woertler K: Tumors and tumor-like lesions of peripheral nerves. Semin Musculoskelet Radiol 14:547-558, 2010

15. Yang F, Chen XX, Wu HL, Zhu JF, Chen Y, Yu LF, Huang $\mathrm{XJ}$ : Sonographic features and diagnosis of peripheral schwannomas. J Clin Ultrasound 45:127-133, 2017 